\title{
Investigating the Mechanism of Shear Fatigue in Reinforced Concrete Beams subjected to Pulsating and Moving Loads using Digital Image Correlation
}

\author{
Benny Suryanto ${ }^{1, *}$, George Staniforth ${ }^{1}$, Jaehwan $\mathrm{Kim}^{1}$, Esayas Gebreyouhannes ${ }^{2}$, Nobuhiro Chijiwa ${ }^{3}$, Chikako \\ Fujiyama ${ }^{4}$, and Peter K. Woodward ${ }^{5}$ \\ ${ }^{1}$ Institute for Infrastructure and Environment, Heriot-Watt University, Edinburgh EH14 4AS, United Kingdom \\ ${ }^{2}$ School of Civil and Environmental Engineering, Addis Ababa University-Addis Ababa Institute of Technology, Addis Ababa \\ 1000 , Ethiopia \\ ${ }^{3}$ Department of Civil and Environmental Engineering, Tokyo Institute of Technology, Tokyo 152-8552, Japan \\ ${ }^{4}$ Department of Civil and Environmental Engineering, Hosei University, Tokyo 162-0843, Japan \\ ${ }^{5}$ School of Civil Engineering, University of Leeds, Leeds LS2 9JT, United Kingdom
}

\begin{abstract}
An experimental investigation on three shear-critical reinforced concrete beams was performed to investigate the mechanism of shear fatigue. The first beam was simply tested to failure under monotonic loading to determine the static capacity, whereas the other two were subjected to repetitive loading below its static capacity to failure. Of these two beams, one was subjected to a stationary pulsating load at midspan while the other was subjected to a step-wise moving load along the span. During each experiment, the crack pattern was monitored throughout using an automated crack mapping employing the digital image correlation technique. The results show that each beam exhibited a unique crack pattern which could be characterised as shear-flexure in nature. It is shown the nature of crack propagation under monotonic loading is dissimilar to that under repetitive loading, especially when the load is not stationary. Moving load is also shown to cause greater damage to the beam than the stationary pulsating load and result in a reduction in fatigue life by almost two orders of magnitude.
\end{abstract}

\section{Introduction}

The construction of High Speed 2 (HS2) has now moved on apace, with the first phase connecting London and the West Midlands from 2026 and the second operating from the West Midlands to Leeds and Manchester from 2033. Along these routes, major infrastructure i.e. tunnels, viaducts and bridges will be constructed in the years to come and reinforced concrete will undoubtedly form an integral part of this major development, particularly if concrete slab track is used.

When a high-speed train travels on a bridge, various internal mechanisms can develop within the main superstructure elements. In the main decks and supporting beams, the primary mechanisms are through internal beam action, with the concrete carrying the compressive stresses and the reinforcement carrying mainly the tensile stresses. Over the lifetime of a bridge, however, the situation is often exacerbated by the inevitable presence of a network of cracks in the concrete $[1,2]$ which can alter the internal stress mechanisms, particularly in regions close to the point load of application. Due to repeated application and constant movement of loading, secondary mechanism (i.e. shear) can dominate locally and become the triggers of a premature failure. A good example would be the punching-type failure commonly observed in highway bridge decks [3] highlighting the fact that shear-fatigue is still a pressing problem faced by practicing engineers.

Traditionally shear fatigue was studied on a reinforced concrete beam, with a pulsating load applied at the centre of span where the moment is maximum. Notable contributions were made by Kesler and Taylor $[4,5]$ who tested a range of reinforced concrete beams with no web reinforcement under varying magnitudes of fatigue load, and Farghaly, Okamura and Ueda [6, 7] who were able to quantify, for the first time, the reduction in the magnitude of shear forces resisted by the concrete under fatigue loading. This was done through the measurement of strains in the shear reinforcement, presented against the number of cycles on a log scale. The finding provided a significant step in understanding the mechanism of shear fatigue and formed the basis of the Japanese design specification. 
Occurring in parallel with this advancement were experiments on full-scale models of bridge decks by Matsui in Japan [8, 9] and 1/6.6-scale models by Perdikaris in the US [10] using a moving-wheel testing rig. They found that a moving load had a more damaging effect than a pulsating load and resulted in a grid-like crack pattern which resembled the crack pattern observed on real bridge decks. It was found that the fatigue life of bridge decks under moving wheel was two to three orders of magnitude lower which is alarming.

To study the underlying mechanisms responsible for the significant reduction in the fatigue life of bridge decks, Maekawa and co-workers [11] developed a computational platform employing the direct-path integration scheme incorporating the time-dependent and fatigue constitutive models, implemented within the multi-directional fixed-crack scheme. Through a parametric study, it was shown that the reduction in the fatigue life of bridge decks is attributed to shear fatigue phenomenon on crack interfaces. The reduction is particularly significant when water is present in the cracks $[1,12]$, which is consistent with the observations by [13]. Gebreyouhannes performed shear fatigue simulations on reinforced concrete beams with and without web reinforcement under both pulsating and moving loads [14]. It was found that the beams subjected to a moving load exhibit a reduction in fatigue life of between 2 and 3 orders of magnitude. It was suggested that the reduction is attributed to reversal of shear stresses and the changing ratio between shear capacity and shear force as the load moves along the span. In a separate study, Fujiyama and Maekawa [15] found that steel-composite reinforced concrete bridge decks also exhibit comparable if not larger fatigue life reduction when subjected to a moving load.

While problems related to shear fatigue have been studied at length in the field of structural engineering and computational mechanics and several premature failures have been reported over the past decades, it is noticed that the majority has been mainly on highway bridges and comparatively less number of incidents has been reported on railway bridges, provided the fact that the former is subjected to more complex load conditions. With regards to high speed train infrastructure, this could be due to the fact that in countries with high seismicity such as Japan, it is not the loads exerted from the train that become the main concern in design, but the forces produced by the earthquakes.

In region of relatively low seismicity such as the UK, however, reinforced concrete structures are generally more lightly reinforced and this would mean that the contribution from the concrete in the web region to the overall member shear capacity becomes more prominent, making the structural member more susceptible to shear fatigue. This could be particularly relevant to large scale concrete infrastructure such as for high-speed railways due to the well-known size-effect phenomenon.

This paper presents an experimental study investigating the mechanism of shear fatigue in reinforced concrete beams subjected to a stationary pulsating and a moving load, and how they compare to an identical beam subjected to a monotonic load from which the design code equations are generally based upon. More specifically, this study employs the low-cost automated crack mapping system, which utilises the digital image correlation (DIC) [16, 17], to provide evidence of crack formation and damage evolution during testing. In this paper, attention is directed to the sequence of crack formation and crack propagation obtained from the DIC technique, the load-deflection responses under loading and the progress of beam deflection during the loading cycles.

\section{Experimental programme}

\subsection{Test specimen}

Three reinforced concrete beams were constructed to nominal dimensions of $155 \mathrm{~mm}$ (width) $\times 300 \mathrm{~mm}$ (depth) $\times 2200 \mathrm{~mm}$ (length). Each beam was reinforced with three deformed longitudinal bars with a nominal diameter of $16 \mathrm{~mm}$, all positioned at an effective depth, $d$, of $257 \mathrm{~mm}$. Each beam was tested under a three-point load on an overall span of $1,800 \mathrm{~mm}$. The schematic of the test setup used to perform the beam tests is displayed in Figure 1, with the summary presented in Table 1.

Beam 1 was subjected to centre-span monotonic loading to failure, Beam 2 to $\sim 78 \%$ of the static capacity of Beams 1 before being subjected to fatigue cycles to failure, and Beam 3 to the same load magnitude but with the load applied in sequence at five locations along the beam i.e. 400, 650, 900, 1150, $1400 \mathrm{~mm}$ from the right support, referred to, respectively, as Load Points 1 to 5 . This load arrangement, described in detail below, was employed to simulate a moving load travelling along the top of the beam from right-to-left-hand side and is referred to as a step-wise moving load.

Based on the proposed test configuration, the shear span-to-effective depth $(a / d)$ ratio for Beams 1 and 2 was evaluated as 3.5, whereas the $a / d$ ratio for Beam 3 varied depending on the location of the applied load: $a / d=1.6$ at Load Points 1 and 5, $a / d=2.5$ at Load Points 2 and 4, and $a / d=3.5$ at Load Point 3. Apart from the $a / d$ ratio, the moment/shear $(\mathrm{M} / \mathrm{V})$ ratio in Beams 3 at the section under the point load also varied depending on the location of the point load, while the $\mathrm{M} / \mathrm{V}$ ratio of the same section in Beams 1 and 2 was constant. For a given test configuration, it can be expected as the $\mathrm{M} / \mathrm{V}$ ratio increases, the contribution from the sectional (longitudinal) forces will increase and the beam becomes less shear critical. Low $\mathrm{M} / \mathrm{V}$ ratios will generally lead to

Table 1. Summary of experimental programme.

\begin{tabular}{|c|c|c|}
\hline Beam & Load & Load location \\
\hline 1 & Monotonic & Centre \\
\hline 2 & Pulsating & Centre \\
\hline 3 & $\begin{array}{l}\text { Step-wise } \\
\text { moving }\end{array}$ & $\begin{array}{l}\text { Five locations at } 250 \mathrm{~mm} \\
\text { spacing as indicated in Fig. } 1\end{array}$ \\
\hline
\end{tabular}



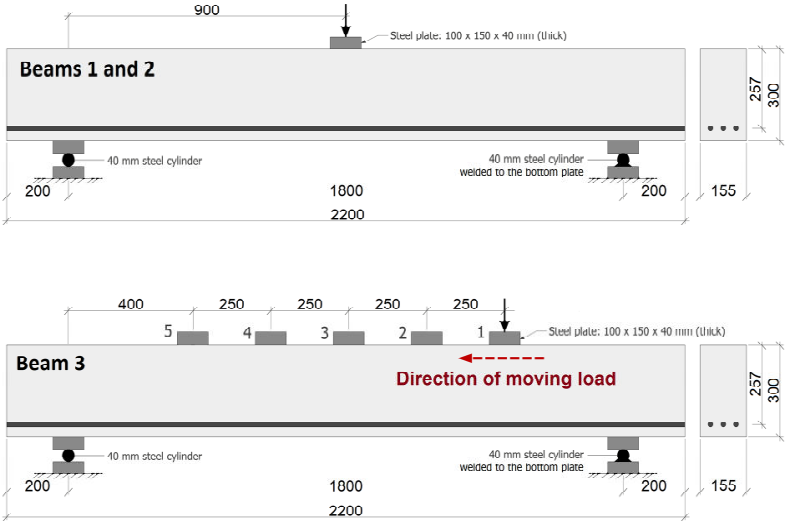

Fig. 1. Beam dimensions and test setup.

the formation of inclined cracks, while high $\mathrm{M} / \mathrm{V}$ ratios will lead to the formation of cracks oriented more vertically. In a beam subjected to a stationary load such as Beams 1 and 2 tested in this study, it can be expected that vertical cracks will develop mostly under the point of load application where the maximum $\mathrm{M} / \mathrm{V}$ ratio is located. This would also apply to Beam 3, although it should be noted that the location of the maximum $\mathrm{M} / \mathrm{V}$ ratio in this beam would not be stationary and would follow the location of the point load as it moves from Load Points 1 to 5. To highlight this aspect, the bending moment and shear diagrams corresponding to the five load points considered are presented in Fig. 2. It is shown that the M/V ratio varies linearly from the support and reaches its maximum value where the moment is maximum. In any case, the $\mathrm{M} / \mathrm{V}$ ratio is equal to the shear span, $a$.

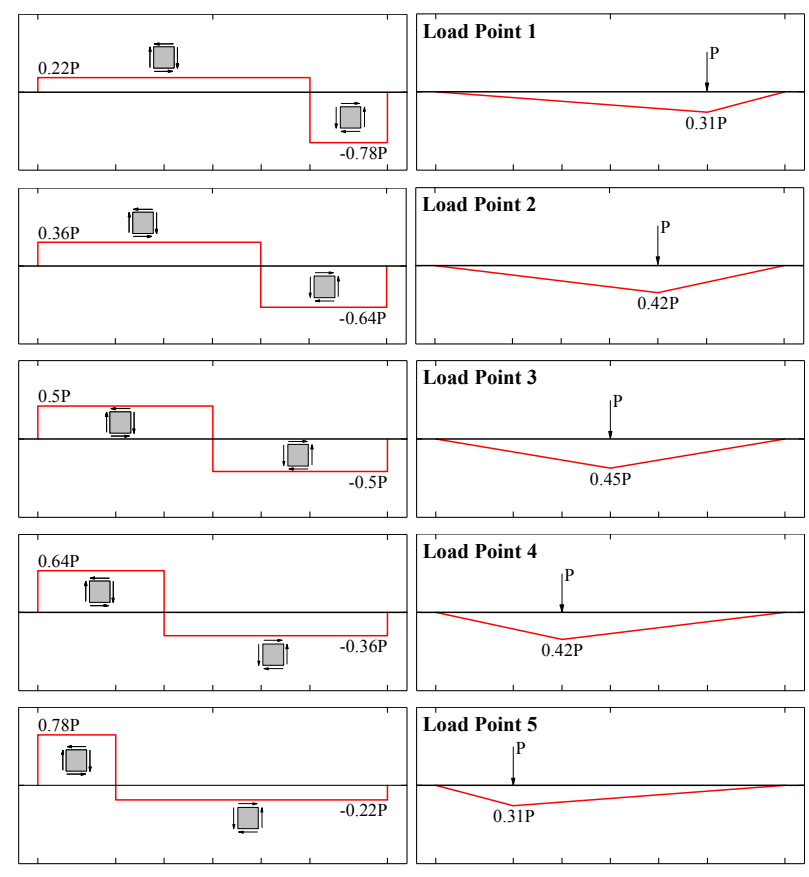

Fig. 2. Shear and bending moment diagrams for Beam 3 during the step-wise moving load cycles. The diagrams under Load Point 3 are also applicable to Beams 1 and 2.
With reference to Fig. 2, it is evident that Beam 3 is subjected to longitudinal and shear stresses of varying magnitudes throughout the load cycles, with the central regions between Load Points 1 and 5 subjected to shear stresses of alternating directions. Due to load movement, the ratio of longitudinal to shear stresses will vary both in time and position along the span, producing complex, non-proportional loading situations. The constant change in the proportion and sign of the stresses would mean that the direction of principal stresses would also constantly change as the load moves from Load Points 1 to 5 . It could be thus anticipated that the concrete in the web region of Beam 3 would be subjected to much more complex stress fields than that of Beams 1 and 2 which is subjected to a stationary load. The behaviour and ensuing crack patterns under these different stress fields are presented and discussed in the later part of this paper.

\subsection{Materials, fabrication and curing}

The concrete used in the beams was C35/45 supplied by a local ready-mix company. The binder comprised a blend of Portland cement CEM I $52.5 \mathrm{~N}$ and ground granulated blast furnace slag at a replacement ratio of 50\%, known as the CEM III/A to BS EN 197-1 [18]. A well-graded basalt aggregate with a maximum size of 20 $\mathrm{mm}$ was used, reflecting common practice in the UK.

On the day of casting, the concrete was cast into a pre-assembled steel framework and compacted using a vibrating rod to facilitate adequate compaction. The top surface of the beams was then levelled with the framework and covered with a polyethylene sheet to minimize moisture loss. After one day, the beams were subsequently wetted on a daily basis for further 6 days and the framework was then removed for the remaining 35 days. During this period, the beams were cured under a polyethylene sheet in a laboratory environment and wetted twice a week to facilitate curing and minimize drying shrinkage-induced cracking. Daily mean laboratory temperature ranged between $6-13^{\circ} \mathrm{C}$. The beams were tested between 42 and 54 days after casting.

\subsection{Test equipment, instrumentation and load protocol}

The tests were conducted using a 2,000 $\mathrm{kN}$ Losenhausen UPS200 servo-hydraulic testing machine, as used in the GRAFT facility [19]. Prior to testing, the centre of each beam was positioned directly below the crosshead of the machine. The beam was then rested on two supports, each comprising: (i) a $40 \mathrm{~mm}$-diameter steel rod sandwiched by two $100 \times 150 \times 40$ (thick) $\mathrm{mm}$ steel plates; and (ii) a $200 \mathrm{~mm}$ high rigid steel base to provide adequate clearance for placing instrumentation under the beam. These steel assemblages were then placed on the rigid steel base of the test machine $(\sim 500 \mathrm{~mm}$ thick), particularly suited for testing specimens with high stiffness as the beams tested in this work.

Prior to testing, the beam was positioned following the setup depicted in Fig. 1. A $300 \mathrm{kN}$ load cell was then placed on a $40 \mathrm{~mm}$ thick steel plate, which was rested 

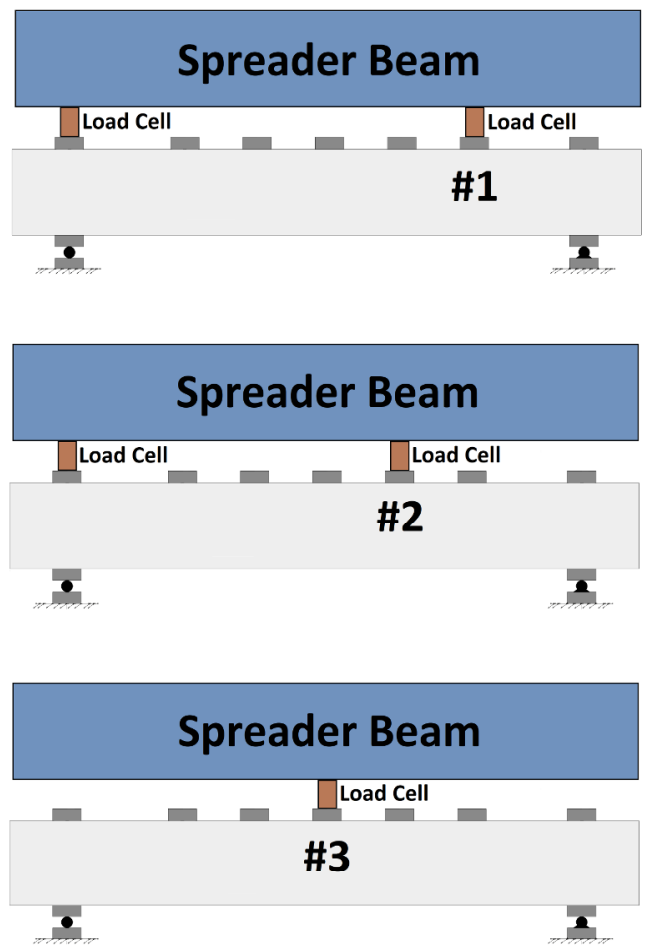

Fig. 3. Schematic of the step-wise moving load protocol employed for Beam 3. Load steps 4 and 5 are the mirror of load steps 2 and 1, respectively. Two load cells were employed in load steps 1, 2, 4 and 5 to maintain stability and equilibrium while only one load cell was employed in load step 3 .

directly on the top centre of the beam. For Beam 1, loading was then performed under a displacement control at a constant rate of $0.2 \mathrm{~mm} / \mathrm{min}$ using a closedloop servo-controlled system. The applied displacement was stopped at $20 \mathrm{kN}$ intervals to visually assess cracking at the rear side of the beam. To monitor beam deflection at the mid-span, a precision linear variable displacement transducer was placed under the beam and aligned vertically under the point load by means of a magnetic holder. Readings for the load cell and transducer were taken using a separate data acquisition system connected to a desktop PC at a rate of $2 \mathrm{~Hz}$.

Beam 2 was tested using the same test setup and loading protocol as above. When the load reached 88 $\mathrm{kN}$, however, the beam was fully unloaded and fatigue loading was then applied under a force control over the load range $15-88 \mathrm{kN}$ at a rate of $70 \mathrm{kN} / \mathrm{min}$. The loading rate was further increased to $350 \mathrm{kN} / \mathrm{min}$ after 100 cycles and subsequently to $700 \mathrm{kN} / \mathrm{min}$ after 500 cycles.

Beam 3 was subjected to a step-wise moving load following the loading protocol displayed in Fig. 3. To perform the moving load, a heavily-stiffened steel section with an overall depth of $400 \mathrm{~mm}$ was employed and clamped to the crosshead of the testing machine, acting as a spreader beam. Below this beam, two $300 \mathrm{kN}$ spherical head load cells were arranged at locations following the schematic depicted in Fig. 3. One full passage comprised five load steps. In Load Step 1, one of the load cells was positioned directly above the left support and the other at Load Point 1, as indicated in Fig. 3. The beam was then loaded to $88 \mathrm{kN}$ before being fully unloaded. The spreader beam was then lifted to allow the load cell on the right to be shifted to Load Point 2 (see Fig. 3). The beam was then subjected to same load cycle of amplitude $88 \mathrm{kN}$. To perform Load Step 3, the spreader beam was first lifted, the sphericalhead load cell on the right was moved to Load Point 3 while the other was taken off temporarily. The beam was then loaded as before. This continued with Load Steps 4 and 5 essentially mirrored Load Steps 2 and 1. To simplify the loading process, seven steel plates with dimensions of 100 (width) $\times 150$ (long) $\times 40$ (thick) $\mathrm{mm}$ were pre-arranged at the locations indicated in Fig. 3; as such, it was only necessary to move the load cells.

\subsection{Automated crack mapping}

In addition to the manual hand-drawn crack mapping, an automated crack mapping was employed using a lowcost digital image correlation system [16]. The system comprised an ordinary $18.4 \mathrm{MP}$ Nikon 1 J4 digital camera and open-source DIC software Ncorr. The camera was mounted on a tripod at a distance $\sim 600 \mathrm{~mm}$ from the front surface of the beam. Images were collected remotely using an Iphone $4 \mathrm{~S}$ via Nikon wireless mobility utility application.

Prior to testing, the beam was painted white and along the full length of the front surface of each beam, random black patterns were created using a dotted-effect black spray paint. This process was straightforward (generally took less than five minutes) and produced a speckle pattern that was of high quality in terms of speckle spacing, size, and randomness (see Fig. 4). A number of LED lights were employed to minimize the shadow from surrounding equipment. At the time of testing, however, only alternating current LED lights were available which introduced some flickering issues. This problem will be addressed in a further study.

The acquired images were processed using an opensource DIC software Ncorr $[16,17,20]$. To this end, the longitudinal strain profiles obtained from the DIC analysis were used to assess the extent of damage during the load cycles. To better compare the extent of cracks at different orientations, it would be ideal to use the principal tensile strains; however, this would require the development of a post-processing extension to the Ncorr which at the time of writing is still under development.

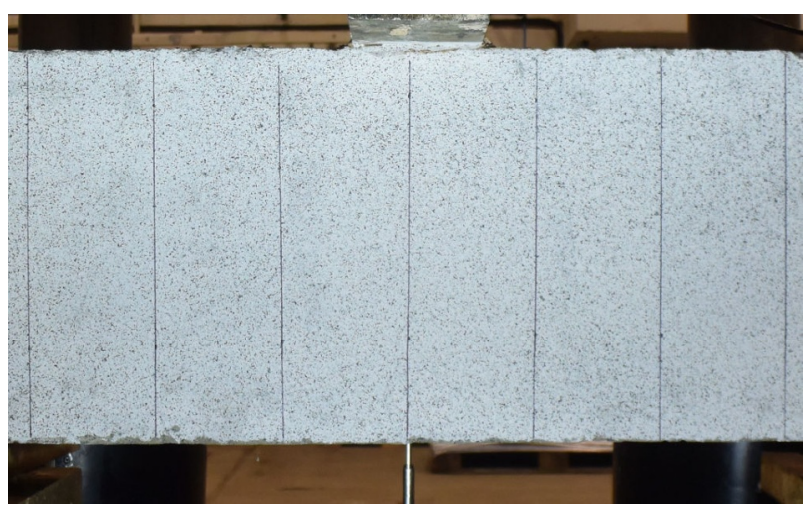

Fig. 4. Random speckled pattern on the front surface. The vertical lines were drawn manually at $100 \mathrm{~mm}$ horizontal spacing and used to determine the exact location of the cracks. 


\section{Test results and discussion}

\subsection{Response under monotonic load}

The load versus mid-span deflection response for Beam 1 subjected to monotonic loading is presented in Fig. 5(a), with the longitudinal strain profiles obtained from the DIC analysis displayed in Fig. 5(b) to provide a full insight into the crack development and propagation as the beam was subjected to loading. The longitudinal strain profiles are presented at seven load stages: the first six correspond to the loads from start to peak (particularly at loads when the test was briefly stopped to allow cracking at the rear side of the beam to be visually assessed) and the last one corresponds to the state of the beam immediately after failure and before the beam was fully unloaded.

It is evident from Fig. 5(a) that the beam displays an initial linear-elastic region, with deviation from linearity occurring from approximately $20 \mathrm{kN}$. This corresponds to a bending moment in the beam of $9 \mathrm{kNm}$ and a tensile stress in the concrete at the bottom surface of the beam of approximately $3.9 \mathrm{MPa}$. At $30 \mathrm{kN}$, two flexural cracks had already developed and extended to half the beam depth (see Fig. 5(b)). As the load increased to 80 $\mathrm{kN}$, additional flexural cracks developed symmetrically about the centre at $120 \mathrm{~mm}$ centres. These cracks extended quickly over half the beam depth, with the cracks located away from the centre experiencing a slight rotation at their tips thereby forming a diagonal crack pattern. Crack propagation thereafter was marginal and the beam response was mainly dominated by the opening of pre-existing cracks. When the load reached $110 \mathrm{kN}$, the beam failed in a brittle manner as the diagonal crack in the left-hand-side quickly propagated to the point of load application and to the support along the bottom reinforcement, resulting in a typical S-shaped failure crack pattern. It is interesting to note from Fig. 5(a) that after first cracking, the beam displayed an approximately linear response, with a post-cracking stiffness of only about $30 \%$ of the initial (uncracked) stiffness signifying a significant loss of member stiffness.

Predictions of several design code equations i.e. BSEN1992 [21], JSCE2007 [22], ACI318-14 [23] and CSA A23.3 [24] for the shear strength of beams containing no shear reinforcement are presented in Fig. 5(a) to provide insights into the experimental data obtained. It is evident from the comparison that all design code shear strength predictions are conservative [25]. The most accurate prediction was shown by the BSEN1992 which had a predicted/observed ratio of 0.98 , while the least accurate prediction was shown by the ACI318-14 which resulted in a predicted/observed ratio of 0.77 . The JSCE design code provided the second most accurate prediction at $89 \mathrm{kN}$, with a predicted/observed ratio of 0.81 . In the analysis, the mean concrete compressive strength was taken as $41.6 \mathrm{MPa}$ and this was converted from the mean cube compressive strength of the concrete which was determined on three $100 \mathrm{~mm}$ cubes at 49 days.

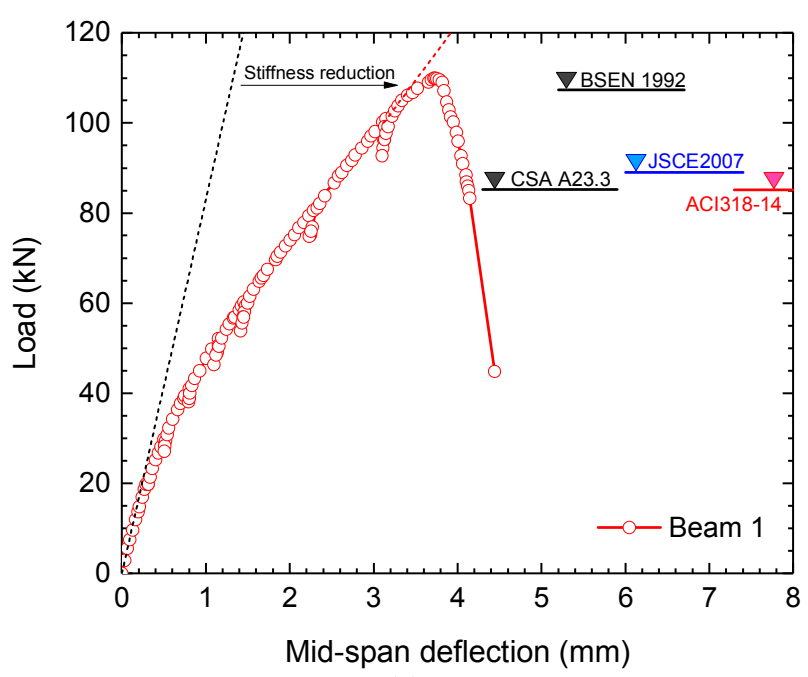

(a)
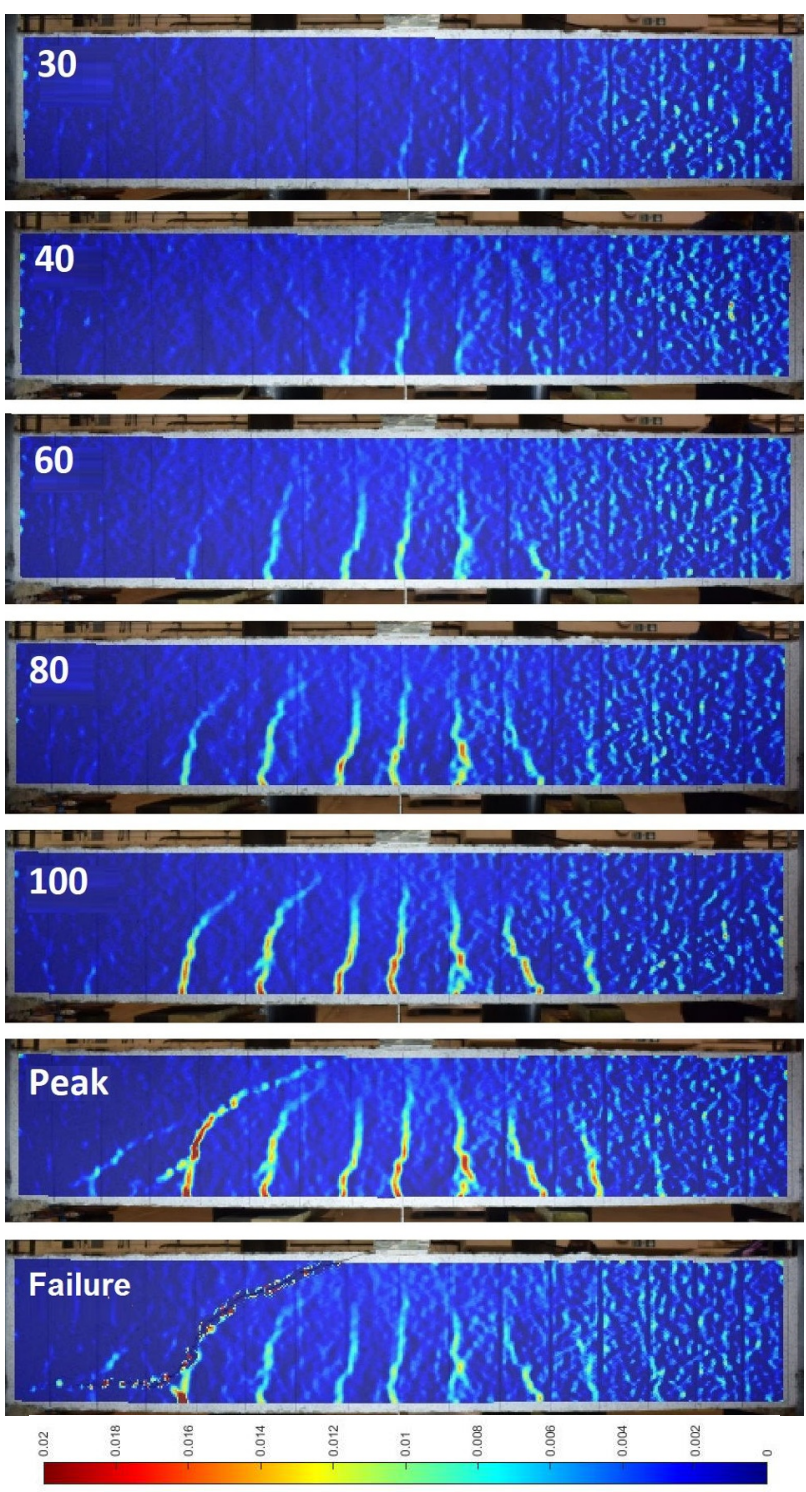

(b)

Fig. 5. (a) Load-deflection response for Beam 1. (b) Longitudinal strain profiles at various load levels (Number: load level in kN; Peak: Peak load; Failure: Immediate after failure). Patches on the right are due to lighting issue. 


\subsection{Response under pulsating load}

The load vs mid-span deflection responses for Beam 2 are displayed in Fig. 6 at selected numbers of load cycles to reduce cluttering. Residual beam deflections were evident as the beam was cycled and partially unloaded to $15 \mathrm{kN}$. The residual deflections were cumulative with increasing load cycles, indicating damage accumulation within the concrete. Occurring in parallel with the damage accumulation is a gradual reduction in overall

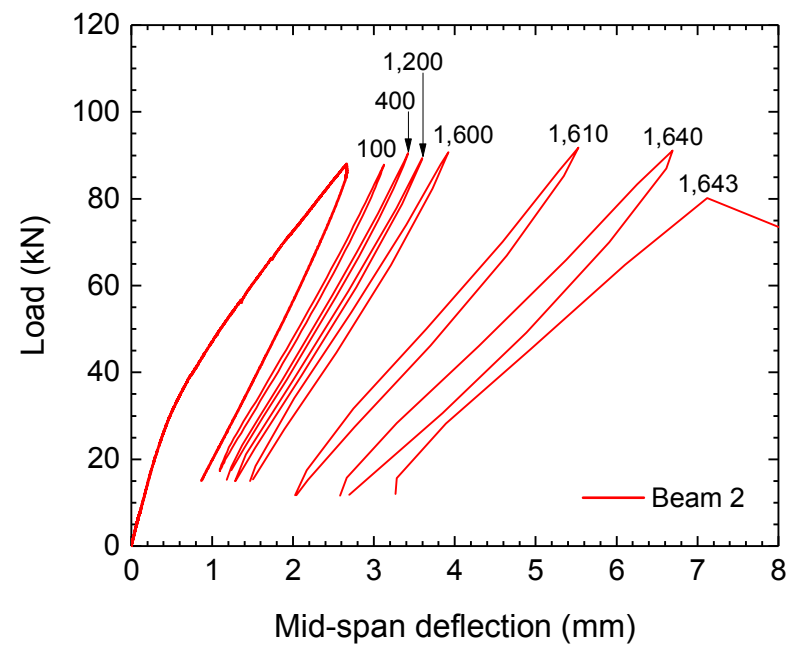

Fig. 6. Load-deflection response for Beam 2 tested under fixed pulsating load applied at the centre span. The number indicates the elapsed number of load cycles.
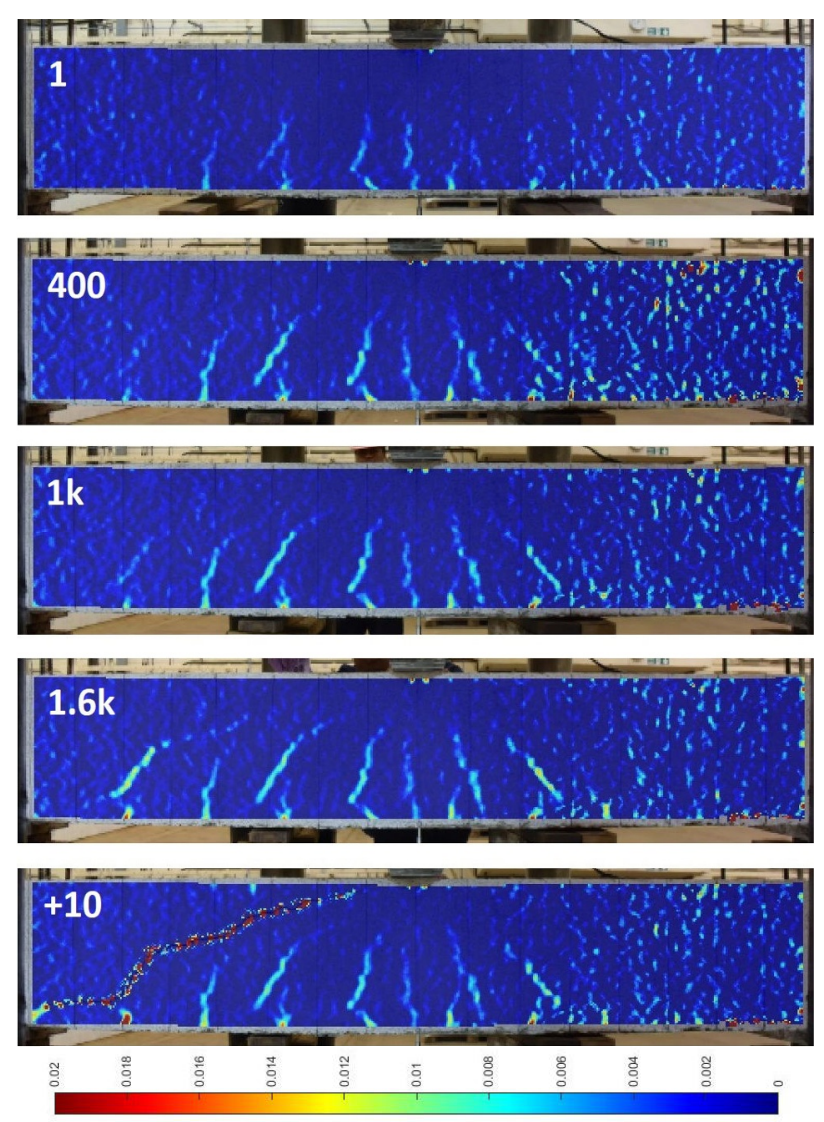

Fig. 7. Calculated longitudinal strain profiles for Beam 2 under pulsating load applied at the centre. The number indicates the elapsed number of cycles. Patches are due to lighting issue. beam stiffness with increasing load cycles (see Fig. 6). This is as a direct result of progressive fracturing and crack propagation within the concrete which are well documented [11-15].

To better illustrate the progress of damage in Beam 2, Figure 7 displays the longitudinal strain profiles obtained after $1,400,1 \mathrm{k}, 1.6 \mathrm{k}$ and $1.61 \mathrm{k}$ cycles. The initial cracking was uniform and predominantly flexural, resembling the crack pattern of Beam 1, although direct comparison is not possible due to unintended variations in the thickness of the base (white) paint and in brightness. Nevertheless, it is interesting to note from Fig. 7 that the inclined cracks located away from the load point were oriented at smaller angles to the horizontal, producing a fan-shaped pattern crack. The extent and prominence of these cracks became more discernible as the beam was subjected up to 400 cycles indicating the direct influence of load cycles (as opposed to load increment in Beam 1). No obvious differences were evident during the subsequent cycles. At $1 \mathrm{k}$ cycles, a new inclined crack had initiated in the web region at the most left-hand-side while the centre region still experiencing greater distress. At 1.6 cycles, this crack had developed into a major S-shaped crack which extended over the web region and quickly propagated over the following 10 cycles to the edge of both the loading plate and the support, causing the beam to fail in shear at 1,643 cycles in a very brittle manner.

\subsection{Response under step-wise moving load}

The load versus mid-span deflection response of Beam 3 at five selected number of passages is presented in Fig. 8 in solid lines, together with the mid-span deflection recorded during the first two loading steps (Points 1 and 2 ) in dotted line for completeness. Although the beam deflection was recorded at five locations under the load points and monitored throughout as the load was moved from Load Points 1 to 5, only the load-deflection relation when the load applied at the centre (Point 3 ) is presented due to the large amount of data acquired from this series of test.

It is clear from Fig. 8 that moving load introduced a far more damaging effect to the concrete, reducing the fatigue life of the beam by almost two orders of magnitude from $1.6 \mathrm{k}+$ cycles to 51 passages only. This is due to the fact that moving load has the effect of constantly changing the principal stress orientation (see Section 2.1) $[2,12,14]$ which, in turn, has the effect of accelerating fracture and damage processes within the concrete. The evidence for this can be seen from the hysteresis at each load cycle presented in Fig. 8. Unlike Beam 2 which exhibits a high degree of elastic hysteresis (as the beam deflects and is then unloaded, it virtually returns to its original deflection thereby forming a closed hysteresis), Beam 3 always exhibits a significant residual deflection upon unloading so as displaying an open hysteresis. This can be attributed to the fact that as the load moves from Load Points 1 to 5 along the beam, it causes shear stresses to develop along the inclined cracks and to change sign as the load passes these cracks [12]. 
As the load moves across any crack, this causes two surfaces of the crack to slide against each other and widen which accelerate damage progression [2, 13, 26]. The change in principal stress directions (see Section 2.1) also makes the pre-existing cracks to propagate in different orientations. For clarity, the longitudinal strain profiles obtained from the DIC analysis is presented in Fig. 9.

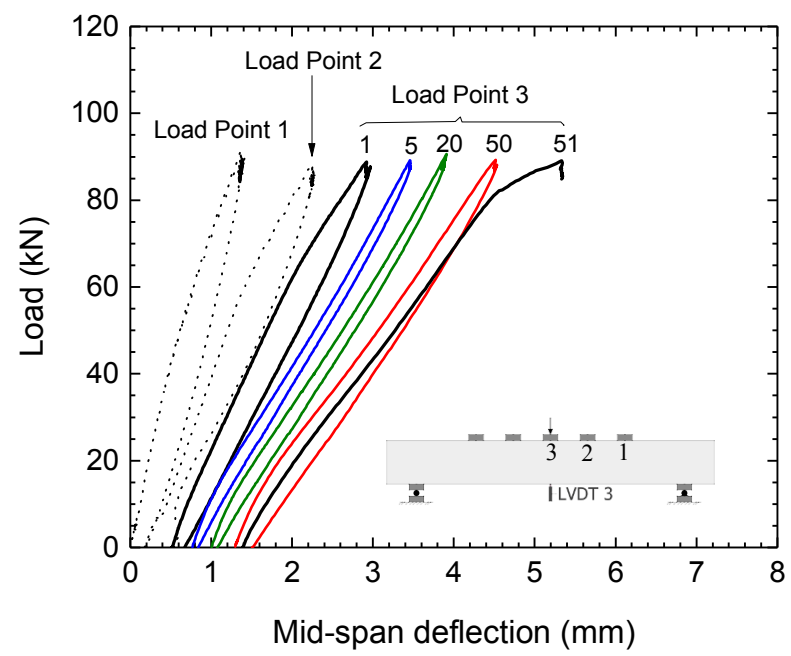

Fig. 8. Load-deflection response for Beam 3 subjected to the proposed step-wise moving load protocol at five locations along the beam. The number indicates the elapsed number of passage (one passage comprises five load steps).
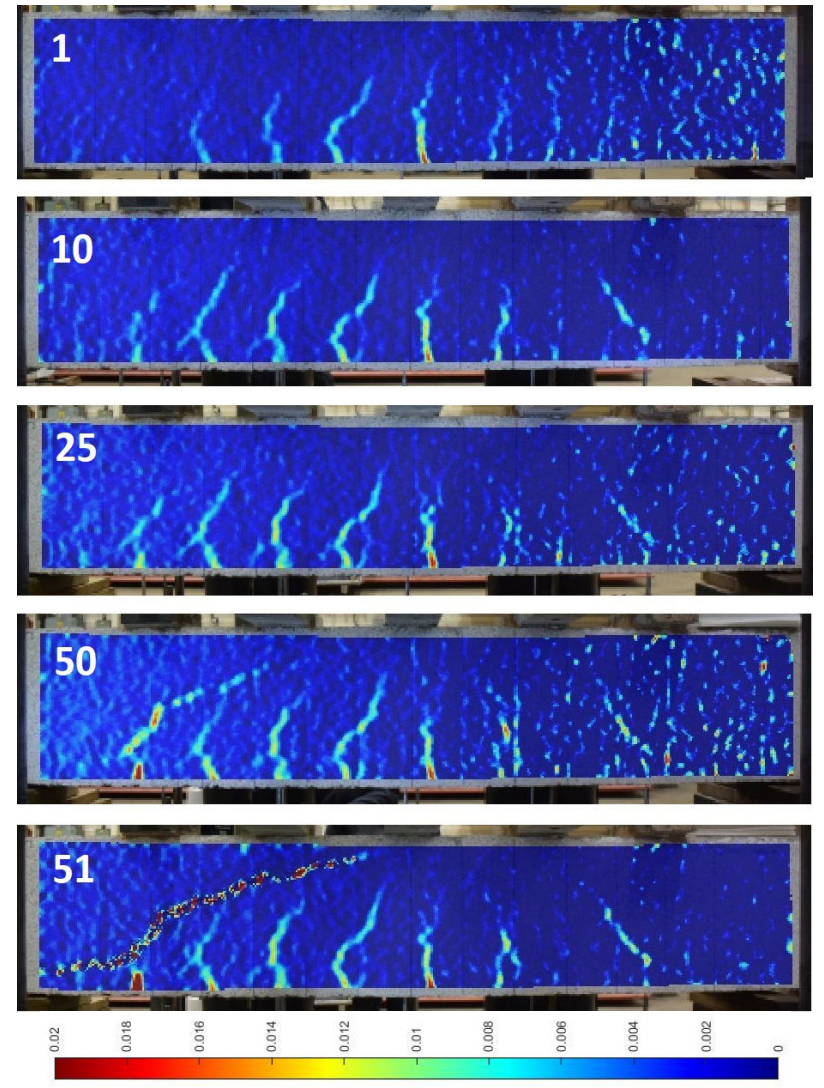

Fig. 9. DIC longitudinal strain profiles for Beam 3 subjected to a step-wise moving load applied at $250 \mathrm{~mm}$ centres. The number indicates the elapsed number of passages. Patches on the right are due to lighting issue.
Overall the crack pattern of Beam 3 displayed in Fig. 9 takes the general form of those shown in Figs. 5 and 7 with respect to the overall pattern and spacing, although the cracks in Beam 3 are found to occur over a wider area. The cracks in Beam 3 are found to develop at early stages of loading; when observed at 10 passages, they had extended over half the beam depth. On closer examination, it is interesting to note that the crack geometry in Beam 3 is rather different. Unlike in Beams 1 and 2 in which the cracks are relatively straight, the cracks in Beam 3 follow a rather wavy path. This is due to the constant change in principal stress direction resulting from the constant change in $\mathrm{M} / \mathrm{V}$ ratios (see Fig. 2) which warrants further detailed investigation (i.e. using a nonlinear finite element analysis). The exact influence of moving load on crack development and propagation will be reported in a future publication.

It is interesting to note from the crack pattern presented in Fig. 9 that the critical crack on the left was an extension of a flexural-shear crack that had developed at initial stages of loading (i.e. before the $10^{\text {th }}$ passage). This suggests that while moving load introduces a far more damaging effect, it leads to the initiation of critical crack at initial stages of loading which progresses quite slowly under repetitive loading. The would imply that if this critical crack could be identified early (i.e. [27, 28]), this could be of practical significance as a means of avoiding undesirable brittle shear failure through direct interventions (i.e. retrofit).

\subsection{Damage progress under fatigue loading}

To provide a better understanding of the damage evolution during load cycles, the upper and lower deflection envelopes recorded from both beams are presented in Fig. 10; for Beam 2, they are plotted against the logarithm of the number of cycles while for Beam 3 against the logarithm of the number of passages. Note that in Beam 2, both load and deflection measurements were undertaken only at the centre span (Load Point 3) whereas in Beam 3 the load was applied at five discrete locations along the beams (Load Points 1-5). While deflection measurements in Beam 3 were undertaken at five locations below the predetermined load points, only the deflection measured at the centre span (Load Point 3) is presented in the figure to enable direct comparison with that of Beam 2.

It is interesting to note from Fig. 10 that the upper deflection envelope of Beam 3 displays notable fluctuations in value showing a successive formation of a triangle-like pattern which merges together as the number of passages increases, due to the logarithmic scale of the horizontal axis. This is as a direct result of the step-wise movement of the load from Points 1 to 5, as indicated in the figure during the first load passage. At each passage, the beam generally reaches its maximum deflection (the peak of the triangle-like pattern) when the load was applied at the centre span (Load Point 3). Increasing load passage is shown to result in a progressive upward movement of the envelope plots indicating that the damage in the beam is progressive 


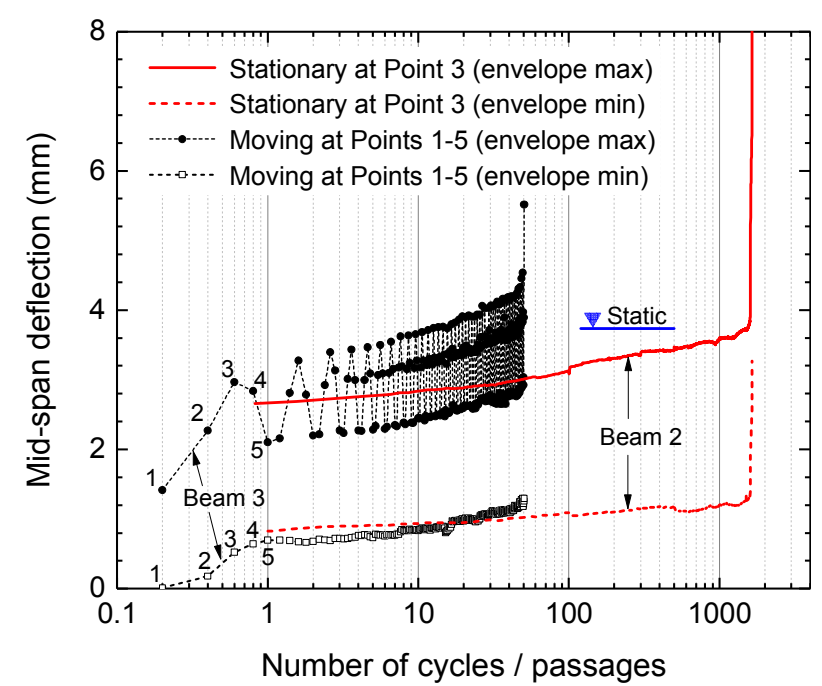

Fig. 10. Comparison of the envelopes of mid-span deflection in Beam 2 subjected to a stationary (pulsating) load and Beam 3 subjected to a step-wise moving load. Numbers 1-5 are the locations of the load point along the beam (see Fig. 2). Load point 3 is located at the centre span.

and accumulative. A significant increase in deflection is seen when the beam reaches $\sim 45$ cycles, corresponding to a mid-span deflection of $\sim 4.3 \mathrm{~mm}$. This quickly increased to $\sim 5.5 \mathrm{~mm}$ over the next five passages before the beam eventually fails in shear (see Figs. 8 and 9). The beam deflection prior to failure is $\sim 1.5$ times that of Beam 1 and this is due to the greater damage experienced by Beam 3 prior to failure (see Fig. 9).

Similar to the trend exhibited by Beam 3, it is apparent from Fig. 10 that Beam 2 displays a steady increase in deflection with increasing number of cycles indicating increasing damage with increasing number of load cycles. However, it is clear that the rate of deflection increase in this beam is generally much lower than that in Beam 3, indicating that the stationary pulsating load introduces a less damaging effect than the step-wise moving load. The evidence of this can be seen from the magnitude of the mid-span deflection itself, with that of Beam 2 always displaying a smaller value than that of Beam 3 even from the first cycle/passage. The rate of deflection increase in Beam 2 rises markedly as the beam has reached $\sim 1.5 \mathrm{k}$ cycles or at a corresponding mid-span deflection of $\sim 3.7 \mathrm{~mm}$ which is comparable to the deflection of Beam 1 at the peak load (as indicated in the Figure) despite the difference in crack patterns (compare Figs. 5 and 7).

Finally, it is of interest to note that direct comparison of the lower envelope plots is not possible due to the fact that Beam 2 was unloaded partially to $\sim 15 \%$ of the static capacity to ensure the stability of the test setup during load cycles, whereas Beam 3 was fully unloaded to allow the spreader beam to be lifted and the load-cells to be repositioned following the diagram shown in Fig. 3. Nonetheless, the lower envelope plots clearly show that the residual deflections upon unloading are also progressive and cumulative, indicating progressive damage of the concrete with increasing number of load cycles/passages and progressive widening of residual crack opening upon full/partial unloading.

\section{Concluding remarks}

Automated crack mapping was employed to provide direct evidence on strain distributions and ensuing crack patterns in shear-critical reinforced concrete beams subjected to three different loading patterns: monotonic, fixed-pulsating and moving load. Attention was particularly directed to the sequence of crack initiation and propagation during the loading, the corresponding load-deflection relation, and the progress of beam deflection under repeated load cycles. The main intention was to provide direct evidence on the mechanisms that lead to the well-known fatigue phenomenon responsible for premature failure in reinforced concrete bridge decks.

The results presented have shown that the step-wise moving load introduced a far more damaging effect to the concrete, reducing shear fatigue life of the beam by almost two orders of magnitude from $1.6 \mathrm{k}+$ cycles to 51 load passages only. The greater damage experienced by the beam subjected to the moving load is confirmed by the greater increase in beam deflection with increasing load cycles/passages. It is shown that the critical crack that may lead to shear failure can initiate well before failure takes place and propagate relatively slowly under repetitive loading. Early crack identification could of practical significance as a means of avoiding brittle shear failure through, for example, direct interventions.

The authors wish to acknowledge the support of the Institute for Infrastructure and Environment at Heriot-Watt University for placing the facilities of the Institute at their disposal. Financial supports from Hosei University and Tokyo Institute of Technology are also gratefully acknowledged.

\section{References}

1. K. Maekawa, T. Ishida, N. Chijiwa, C. Fujiyama, ASCE J. Mater. Civil Eng. 27, 2, A4014003 (2013)

2. B. Suryanto, K. Nagai, K. Maekawa, ACI Mater. J. 107, 5, 450-460 (2010)

3. J. Rhee, H. Kim, C. Ock, J. Choi, KSCE J. Civ. Eng. 22, 2, 613-621 (2018)

4. T.S. Chang, C.E. Kesler, ACI J. 55, 8, 245-254 (1958)

5. R. Taylor, ACI J. 55, 14, 1011-1015 (1959)

6. S.A. Farghaly, PhD thesis (University of Tokyo, 1979)

7. H. Okamura, S.A. Farghaly, T. Ueda, Proc. JSCE 308, 109-122 (1981)

8. S. Matsui, $\mathrm{PhD}$ thesis (Osaka University, 1984)

9. S. Matsui, Proc. JCI 9, 2, 627-632 (1987)

10. P.C. Perdikaris, S. Beim, ASCE J. Struct. Eng. 114, 3, 591-607 (1988)

11. K. Maekawa, K. Toongoenthong, E. Gebreyouhannes, T. Kishi, J. Adv. Concr. Technol. 4, 1, 159-177 (2006)

12. K. Maekawa, E. Gebreyouhannes, T. Mishima, X. An, J. Adv. Concr. Technol. 4, 3, 445-457 (2006) 
13. E. Gebreyouhannes, T. Kishi, K. Maekawa, J. Adv. Concr. Technol. 6, 2, 365-376 (2008)

14. E. Gebreyouhannes, N. Chijiwa, C. Fujiyama, K. Maekawa, J. Adv. Concr. Technol. 6, 1, 215-226 (2008)

15. C. Fujiyama, K. Maekawa, J. Adv. Concr. Technol. 9, 2, 193-204 (2011)

16. B. Suryanto, A. Tambusay, P. Suprobo, Civ. Eng. Dimension 19, 2, 93-98 (2017)

17. A. Tambusay, B. Suryanto, P. Suprobo, Int. J. Adv. Sci. Eng. Inf. Technol. 8, 2, 573-578 (2018)

18. British Standard Institution (BSI), BS EN 1971:2011, BSI, London (2011)

19. P.K. Woodward, J. Kennedy, O. Laghrouche, D.P. Connolly, G. Medero, Transport. Geotech. 1, 4, 214224 (2014)

20. J. Blaber, B. Adair, A. Antoniou, Exp. Mech. 55, 6, 1105-1122 (2015)

21. British Standard Institution (BSI), BSEN 1992-11:2004+A1:2014, BSI, London (2004)

22. Japan Society of Civil Engineers (JSCE), JSCE Guidelines for Concrete 15, JSCE, Tokyo (2010)

23. American Concrete Institute (ACI), ACI318-14, ACI (2014)

24. Canadian Standards Association (CSA), A23.3-14, CSA (2014)

25. B. Suryanto, R. Morgan, A.L. Han, Civ. Eng. Dimension 18, 1, 16-24 (2016)

26. B. Suryanto, K. Nagai, K. Maekawa, J. Adv. Concr. Technol. 8, 3, 315-326 (2010)

27. K. Maekawa, X. Zhu, N. Chijiwa, S. Tanabe, J. Adv. Concr. Technol. 14, 5, 183-204 (2016)

28. E. Fathalla, Y. Tanaka, K. Maekawa, Eng. Struct. 171, 602-616 (2018) 